\title{
Consecuencias económicas en la vida real de la inmunoterapia con alérgenos en asma, rinitis y dermatitis
}

\author{
Jorge Sánchez, ${ }^{1-3}$ Andrés Sánchez, ${ }^{1-4}$ Ricardo Cardona ${ }^{1}$
}

\begin{abstract}
Background: Allergen immunotherapy has proved effective in the treatment of various allergies, but whether the clinical benefits outweigh the economic costs has been little evaluated.

Objective: To explore the economic consequences of immunotherapy in asthma, rhinitis and dermatitis.

Methods: Descriptive study. Patients with at least 18 months' follow-up were selected, whose medical history allowed evaluation of drugs received, manner of use, check-ups, treatment changes, reasons for these, and so on. Patients were classified according to whether they received drug treatment + immunotherapy (active group) or drug therapy alone (control group).

Results: 1848 patients were included: 648 in the active group and 1200 in the control. Immunotherapy increased the cost of treatment during the first few months, but after 9 to 12 months drug treatment decreased significantly compared to the control group. In patients with asthma or various diseases, immunotherapy reduced costs of treatment $(p<0.05)$. The active group saw fewer relapses at 18 months $(p<0.05)$.

Conclusion: Allergen immunotherapy allows the sustained reduction in drug treatment, with medium-term financial savings for the patient and the health system.
\end{abstract}

Keywords: Asthma; Cost-benefit analysis; Dermatitis; Immunotherapy; Pharmacotherapy; Rhinitis

Este artículo debe citarse como: Sánchez J, Sánchez A, Cardona R. Consecuencias económicas en la vida real de la inmunoterapia con alérgenos en asma, rinitis y dermatitis. Rev Alerg Mex. 2016;63(4):323-333

\footnotetext{
${ }^{1}$ Universidad de Antioquia, Grupo de Alergología Clínica y Experimental, IPS Universitaria. Medellín, Antioquia, Colombia.

${ }^{2}$ Fundación para el Desarrollo de las Ciencias Médicas y Biológicas. Cartagena, Colombia.

${ }^{3}$ Universidad de Cartagena, Instituto de investigaciones Inmunológicas, Grupo de Alergología Experimental e Inmunogenética. Cartagena, Colombia.

${ }^{4}$ Corporación Universitaria Rafael Núñez. Cartagena, Colombia.
}

Correspondencia: Jorge Sánchez. jotamsc@yahoo.com

Recibido: 2016-01-17

Aceptado: 2016-03-24

(c) $(1)(3)$ 


\title{
Resumen
}

Antecedentes: La inmunoterapia con alérgenos ha demostrado ser efectiva en el tratamiento de diversas alergias, pero poco se ha evaluado si los beneficios clínicos compensan los costos económicos.

Objetivo: Explorar las consecuencias económicas de la inmunoterapia en asma, rinitis y dermatitis. Métodos: Estudio retrospectivo analítico. Se seleccionaron pacientes con seguimiento por al menos 18 meses, cuya historia clínica permitiera evaluar los medicamentos recibidos, forma de uso, citas de control, cambios en el tratamiento, motivos de estos, etcétera. Los pacientes fueron clasificados de acuerdo a si recibieron tratamiento farmacológico + inmunoterapia (grupo activo) o solo tratamiento farmacológico (grupo control).

Resultados: 1848 pacientes fueron incluidos: 648 en el grupo activo y 1200 en el control. La inmunoterapia aumentó el costo del tratamiento durante los primeros meses, pero después de 9 a 12 meses disminuyó significativamente el tratamiento farmacológico en comparación con el grupo control. En los pacientes con asma o varias enfermedades, la inmunoterapia redujo los costos del tratamiento ( $p<0.05)$. En el grupo activo se observó menos recaídas a los 18 meses $(p<0.05)$.

Conclusión: La inmunoterapia con alérgenos permite la reducción sostenida del tratamiento farmacológico, con ahorro económico a mediano plazo para el paciente y el sistema de salud.

Palabras clave: Asma; Análisis costo-beneficio; Dermatitis; Farmacoterapia; Inmunoterapia; Rinitis

\author{
Abreviaturas y siglas \\ GHIPS, Gestión Hospitalaria IPS \\ $\lg \mathrm{E}$, inmunoglobulina $\mathrm{E}$ \\ IPS, Instituto Prestador de Salud
}

\section{Antecedentes}

Las alergias comprenden un conjunto de enfermedades altamente prevalentes como el asma, la rinitis y la dermatitis, las cuales frecuentemente se presentan de forma simultánea. Cuando los procesos alérgicos no son controlados, pueden dejar secuelas limitantes o poner en peligro la vida de los pacientes. Además del alto costo psicosomático y social, las alergias tienen un alto costo económico que es asumido por el paciente, su familia y el sistema de salud. ${ }^{1}$

Por lo general, el manejo de las alergias incluye tres modalidades:

- Las medidas de evitación que consisten en la identificación y separación de los detonantes ambientales causantes de los síntomas.

- La farmacoterapia.

- La inmunoterapia, cuando se demuestra que los síntomas son causados por una respuesta IgE específica.
De las tres, solo la última tiene un efecto benéfico que perdura años después de terminar el tratamiento, ${ }^{2,3}$ y permite una reducción significativa (en ocasiones total) del tratamiento antiinflamatorio. ${ }^{4}$ Por estos y otros efectos, la Organización Mundial de la Salud la considera la piedra angular del tratamiento de las enfermedades alérgicas y la denomina "vacuna para las alergias", por sus efectos positivos y preventivos, ya que el control inmunomodulador en la rinitis ha demostrado evitar el desarrollo del asma y el agravamiento de los síntomas respiratorios. ${ }^{5,6}$

Actualmente, en buena parte de los países latinoamericanos se dispone de estudios epidemiológicos que han identificado las principales fuentes de alérgenos asociados con síntomas alérgicos, ${ }^{7-9}$ por lo que la inmunoterapia es una opción terapéutica viable en la región, si bien es necesario considerar las preferencias del paciente ${ }^{10}$ y los costos adicionales que genera. De ahí que surja la interrogante de si los beneficios obtenidos de la inmunoterapia justifican 
el esfuerzo económico adicional para el paciente y el sistema de salud.

En el presente artículo presentamos el análisis de los efectos de la inmunoterapia en cuanto a los costos directos en el tratamiento farmacológico y la atención médica en pacientes con asma, rinitis o dermatitis.

\section{Métodos}

Estudio analítico retrospectivo, realizado en un centro de salud en Medellín, Colombia, en el que se incluyeron pacientes que asistieron a consulta entre 2006 y 2013 y que tuvieron seguimiento hasta enero de 2015.

Fueron seleccionados pacientes con diagnóstico de asma, rinitis o dermatitis, con seguimiento de al menos 18 meses en el centro de salud por un médico especialista en alergología, dermatología, otorrinolaringología o neumología. Fueron excluidos los pacientes con información insuficiente respecto al tratamiento de las enfermedades estudiadas o con comorbilidades crónicas que pudieran influir. Se organizaron en dos grupos: activo y control, de acuerdo a si recibieron o no inmunoterapia. Loso criterios de exclusión fueron no contar con información adecuada respecto a dosificación, motivo del cambio de medicación (en caso de ocurrir), forma de uso (adherencia), frecuencia y regularidad.

Aunque diversos especialistas médicos pueden sugerir la inmunoterapia, en este estudio los pacientes la recibieron por indicación final del alergólogo tratante, dado que en Colombia es aplicada por los alergólogos.

\section{Base de datos}

Los datos fueron obtenidos de un centro de referencia (IPS Universitaria, Universidad de Antioquia), el cual tiene, además del Servicio de Alergología, centros de salud que ofrecen atención en neumología, dermatología y otorrinolaringología. En el sistema electrónico de los expedientes clínicos de la institución (Sistema de Gestión Hospitalaria IPS, GHIPS) quedan guardados los datos de cada usuario, los códigos de los diagnósticos y medicamentos prescritos en la consulta, así como otros datos referentes al manejo del paciente. Por ello, la fuente de información para el presente estudio fue el GHIPS.

Se incluyeron pacientes mayores de 3 años que hubieran asistido a la IPS y a quienes se les hubiera diagnosticado asma, rinitis o dermatitis, con al me- nos seguimiento médico en la IPS durante 18 meses $\mathrm{y}$ tres citas de seguimiento:

- Cita 1, primera cita.

- Cita 2, 3 a 6 meses.

- Cita 3, 7 a 12 meses

- Cita 4,13 a 18 meses.

La historia clínica electrónica debía contar con suficiente información para evaluar los medicamentos recibidos y los cambios en el tiempo, adherencia, dosis, suspensión, esquema de uso y motivos de los cambios. Los pacientes podían tener una o más de las enfermedades estudiadas, en ese caso ingresaron en el análisis de cada una de las enfermedades que tuvieran.

\section{Definición de los grupos}

Fueron seleccionados los registros de los pacientes diagnosticados en el centro entre 2006 y 2013. Los pacientes que además del tratamiento farmacológico recibieron inmunoterapia fueron integrados al grupo activo y quienes no recibieron inmunoterapia formaron el grupo control, que solo recibió farmacoterapia.

Para la selección del grupo control se seleccionó a todos los pacientes no expuestos a la inmunoterapia que cumplieron los criterios de selección durante el periodo de estudio, pero se tuvo en cuenta que al menos un paciente del grupo control por cada paciente del grupo activo tuviera una concordancia en las siguientes variables: datos demográficos (edad y sexo), momento diagnóstico de asma, rinitis y dermatitis con diferencia menor $a \pm 6$ meses, periodo de seguimiento y comorbilidades alérgicas.

Para algunas variables demográficas se empleó mediana y rango intercuartílico como medida de dispersión. Se utilizó el Índice de Comorbilidad de Charlson $^{11}$ (originalmente diseñado para predecir el riesgo de mortalidad en pacientes hospitalizados) para excluir a los pacientes con comorbilidades no alérgicas graves que podían aumentar los costos por causas diferentes a las enfermedades estudiadas, así como la respuesta clínica al tratamiento.

Para cada enfermedad se formaron subgrupos dependiendo de la modalidad terapéutica.

\section{Medición de los costos}

Para la evaluación de los costos y la eficacia se consideró el tratamiento recibido al momento del diagnóstico y durante el seguimiento, al igual que el número 
de citas médicas relacionadas con las enfermedades alérgicas. Se cuantificó la dosis, la frecuencia de uso y las concentraciones de los fármacos. De las variables de costos se obtuvo la mediana y el rango como medida de dispersión. Idealmente a cada paciente se le debía asignar el valor exacto del costo de su tratamiento, sin embargo, por no disponer de la información individual, para cada molécula utilizada en la farmacoterapia se obtuvo el promedio del costo de las diferentes marcas disponibles y se consideraron las fluctuaciones en el precio al público en el año evaluado.

Para determinar el valor ponderativo de cada molécula se tomaron en cuenta las marcas más frecuentemente suministradas por el sistema de salud del país. De igual forma se incluyó el valor por periodo de las citas médicas de control.

Debido a que los pacientes pueden tener múltiples enfermedades (asma, rinitis o dermatitis), se evaluó el costo del tratamiento promedio en la primera y última cita del seguimiento, entre los grupos activo y control. El resultado de estas variables se presentó como el promedio en dólares de acuerdo con la tasa de cambio ponderativo durante el periodo de uso ( $\$ 1$ dólar estadounidense $=\$ 2.077$ pesos colombianos).

\section{Consideraciones éticas}

La información obtenida se ajustó estrictamente a las directrices internacionales de la Declaración de Helsinki, cuidando la privacidad de los pacientes. Se obtuvo autorización del comité de ética de la IPS Universitaria.

\section{Análisis estadístico}

En el estudio se evalúo la eficiencia de la inmunoterapia en el tratamiento del asma, la rinitis y la dermatitis. Las variables estudiadas como desenlace primario fueron la reducción en costos de cada enfermedad por separado y el costo por paciente, independientemente del número de enfermedades que tuviera. También se evaluó la suspensión o reducción en el tratamiento recibido entre los grupos activo y control. Los análisis se efectuaron con el programa SPSS versión 21. Las características generales de los pacientes y los resultados se expresaron en porcentajes de frecuencia o en números absolutos. Los costos evaluados estuvieron en relación con el tratamiento utilizado en cada enfermedad, por lo que se compararon los grupos activo y control y de igual forma se integraron subgrupos de acuerdo con los costos de cada enfermedad.

Con las pruebas de Shapiro-Wilk y Kolmogorov-Smirnov se evaluó la distribución y el supuesto de normalidad de las variables de costo y ahorro relacionado (dosis, concentraciones, número de asistencia a centros de salud). Los datos recolectados para las variables de costos y ahorro relacionado no tuvieron distribución normal y se desviaron a la derecha debido a que algunos pacientes sobreutilizaron los servicios de salud y, en consecuencia, los costos se alejaron de la media.

Para corregir los sesgos secundarios a la distribución se utilizó un modelo de regresión lineal generalizada para comparar los costos de atención de los pacientes en cada periodo evaluado con diagnóstico de asma, rinitis o dermatitis que posteriormente recibieron inmunoterapia y fueron emparejados con los de pacientes control que no lo hicieron. Con este modelo también se compararon los ahorros de costos relacionados con la inmunoterapia durante los 18 meses de seguimiento y se analizaron los subgrupos conforme a las enfermedades incluidas.

De acuerdo con la variable evaluada se empleó chi cuadrada para las variables dicotómicas (sí o no en la reducción de la concentración de la medicación y en el costo del tratamiento), regresión logística (variables categóricas reducción de costo, reducción de medicación), grupo (activo versus control) y correlación (costo neto versus concentración de medicación). Algunas variables fueron tratadas de forma continua o categórica de acuerdo con el análisis realizado (ejemplo, análisis de chi cuadrada para sí o no en la reducción del costo y análisis de correlación para valor neto de la reducción). Cuando fue pertinente se hizo análisis de regresión logística multivariada, con ajuste por edad y sexo. Se consideró significativo un valor de $\mathrm{p}<0.05$.

\section{Resultados}

\section{Características de la población}

Se revisaron 11498 ingresos, de los cuales 1848 pacientes cumplieron con los criterios de selección (Figura 1). Entre las comorbilidades que motivaron la exclusión de 400 pacientes estuvieron las enfermedades coronarias no controladas (35\%), cerebrovasculares $(30 \%)$ y otras $(25 \%)$. El rango de edad fue de 3 a 69 años (promedio 21 años); $52 \%$ era del 
sexo femenino, $37 \%$ tenía dos o más de las enfermedades estudiadas, tanto en el grupo control $(n=447)$ como en el activo $(n=244)$, que fue incluido en cada uno los grupos correspondientes cuando el análisis se hizo por enfermedades. En la Figura 1 se presentan los grupos de acuerdo con la enfermedad tomando en cuenta la media y los rangos de edad. Al evaluar los grupos de acuerdo con la mediana y los percentiles 25-75 encontramos los siguientes valores: rinitis en el grupo activo 19 (6-63) y en el control 20 (6-62); asma en el grupo activo 18 (6-62); dermatitis en el grupo activo 15 (5-50) y en el control 17 (5-54).

\section{Costo del tratamiento por enfermedad}

En la cita 1, el grupo activo tuvo un gasto mayor en el tratamiento que el grupo control para las tres enfermedades (Cuadro 1). Este costo adicional estuvo directamente ligado a la inmunoterapia, que representó $17 \%$ del costo del tratamiento en el grupo activo con asma, $35 \%$ con rinitis y $18 \%$ con dermatitis.

Durante el seguimiento, todos los grupos tuvieron una reducción en los costos del tratamiento debido a la reducción de las dosis o la suspensión de medicamentos, la cual fue mayor en las citas 3 y

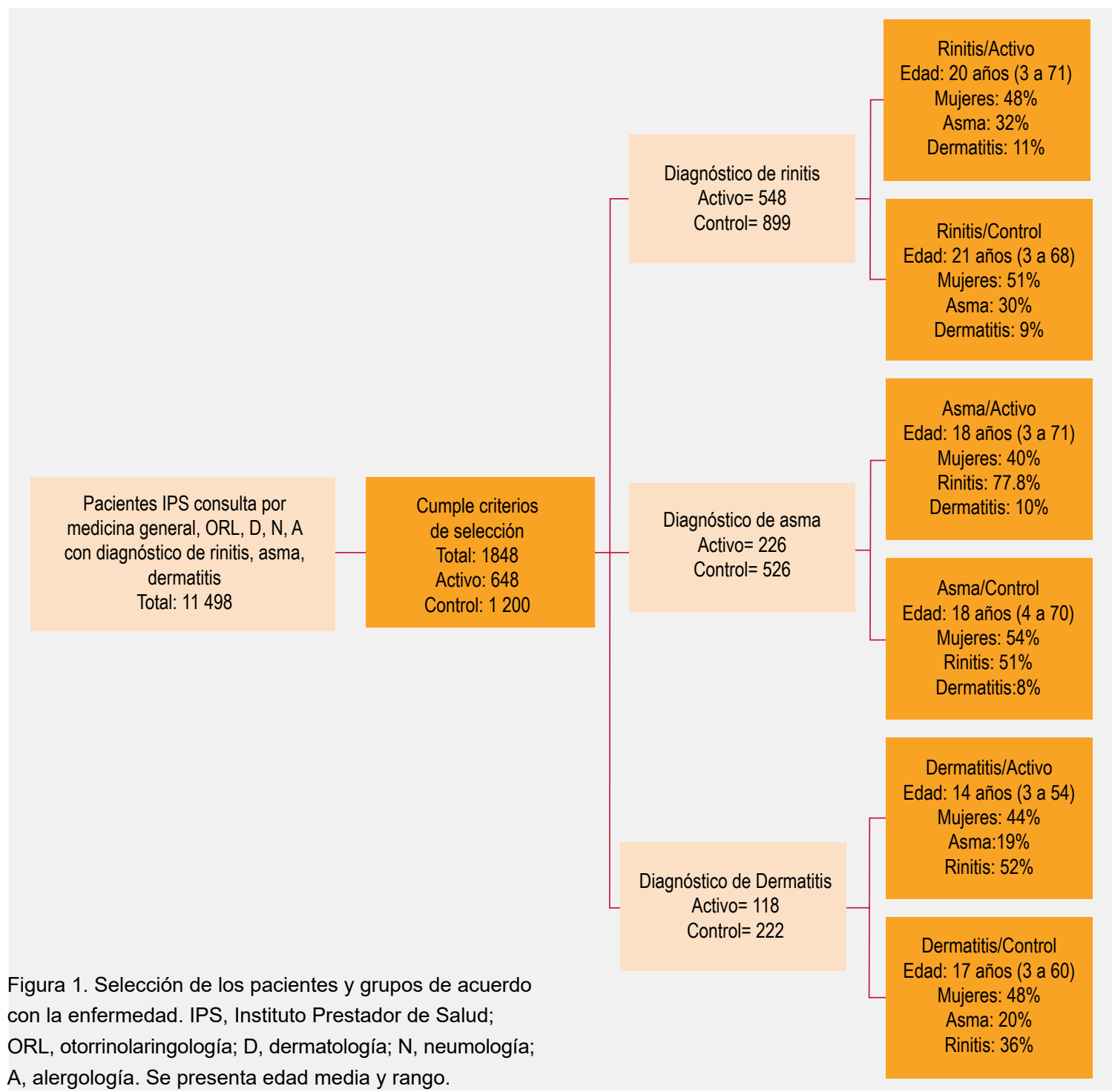


4 en el grupo activo para cada enfermedad. Para la rinitis y la dermatitis, el gasto neto por periodo fue mayor que en los grupos control de cada enfermedad hasta la cita 3; posteriormente, la diferencia no fue estadísticamente significativa. Para el grupo con asma, la reducción en los costos luego de 12 meses del tratamiento fue significativamente mayor en el grupo activo $(\mathrm{p}<0.05)$.

\section{Reducción de la medicación durante el segui-} miento

De acuerdo con la respuesta clínica de cada paciente al tratamiento instaurado en la cita previa, el médico tratante ajustaba el tratamiento en la cita siguiente (aumentando la medicación, dejándola igual o reduciéndola). Todos los grupos presentaron reducción en el tratamiento farmacológico en la cita 2 (Figura 2). En los grupos con inmunoterapia, la reducción de los medicamentos fue sostenida en las tres citas de seguimiento, mientras que en los grupos control, la reducción fue menor y en la cita 3 fue necesario aumentar el tratamiento farmacológico en un grupo. Esta diferencia entre los grupos control y activo fue más evidente en la cita 4.

Al evaluar mediante regresión logística multivariable si la edad ( $\leq 14$ años o $>14$ años) influía en los resultados, se observó que los pacientes $\leq 14$ años con enfermedades respiratorias tenían una reducción sostenida mayor del tratamiento $(\mathrm{p}=0.04)$ independientemente del sexo.

\section{Gasto global por paciente}

Un 37\% de los pacientes presentó múltiples enfermedades (asma, rinitis o dermatitis). Al inicio del estudio, el grupo activo tenía un sobrecosto frente al grupo control de 33 a $23 \%$ debido a la inmunoterapia. Al final de la evaluación, la reducción de los medicamentos y del número de citas fue significativa en el grupo activo comparado con el grupo control (Figura 3). Al comparar la suspensión de medicamentos (Figura 4) en el último periodo por más de 3 meses debido a un buen control y sin recaídas, la reducción fue mayor en el grupo activo en comparación con el control para rinitis ( $9 \%$ versus $5 \%$ ), asma ( $8 \%$ versus $4 \%$ ) y dermatitis (2 versus $1 \%$ ), siendo estadísticamente significativa para las enfermedades respiratorias. Dado que numerosos pacientes padecían múltiples enfermedades, se compararon los grupos activo y control independientemente de las enfermedades base; se observó una suspensión mayor del tratamiento farmacológico en el grupo activo (12\% versus $6 \%)$.

\section{Discusión}

La farmacoterapia es ampliamente usada en asma, rinitis y dermatitis. En la mayoría de las ocasiones suele ser efectiva para controlar los síntomas, sin embargo, no cambia el curso de las alergias y no previene el aumento en su prevalencia. La identificación de las fuentes causantes de los síntomas permite implementar medidas encaminadas a reducir la exposición a los

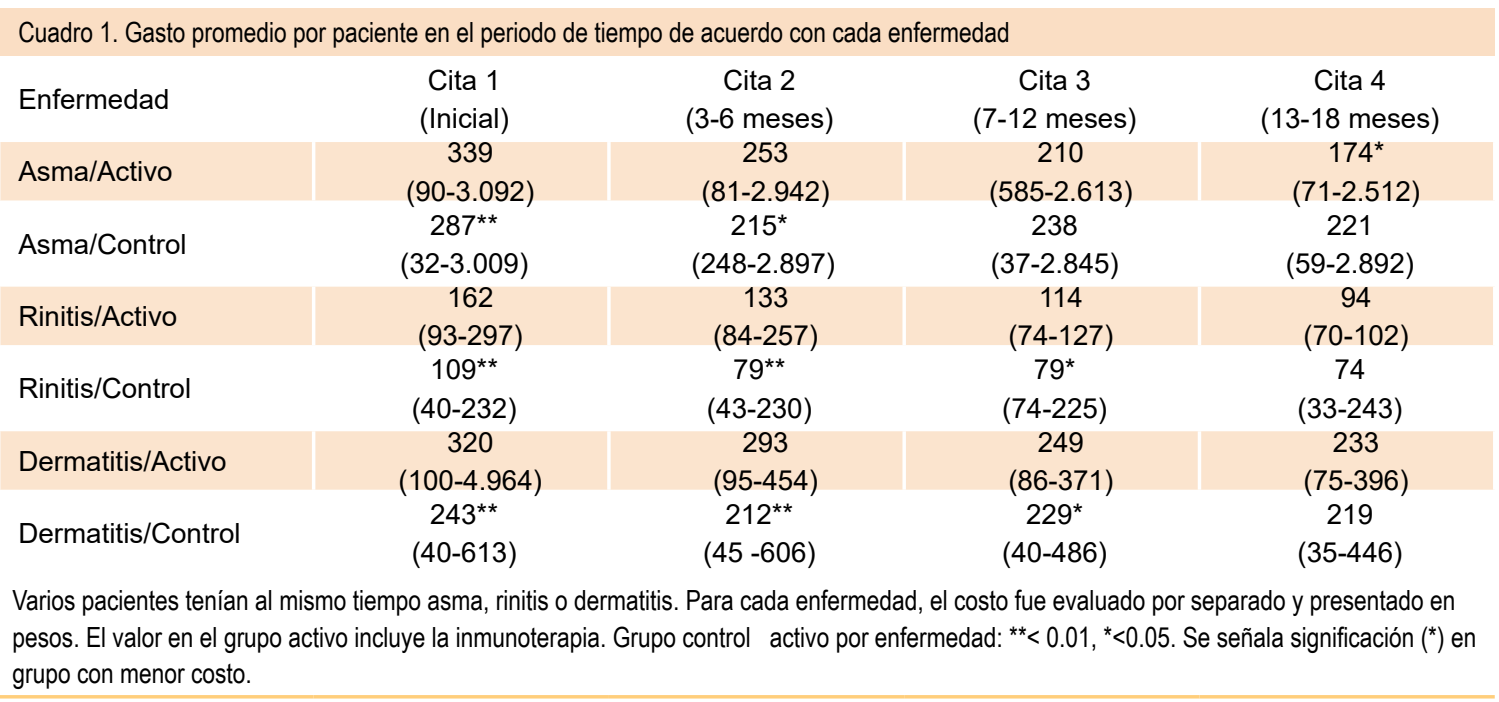




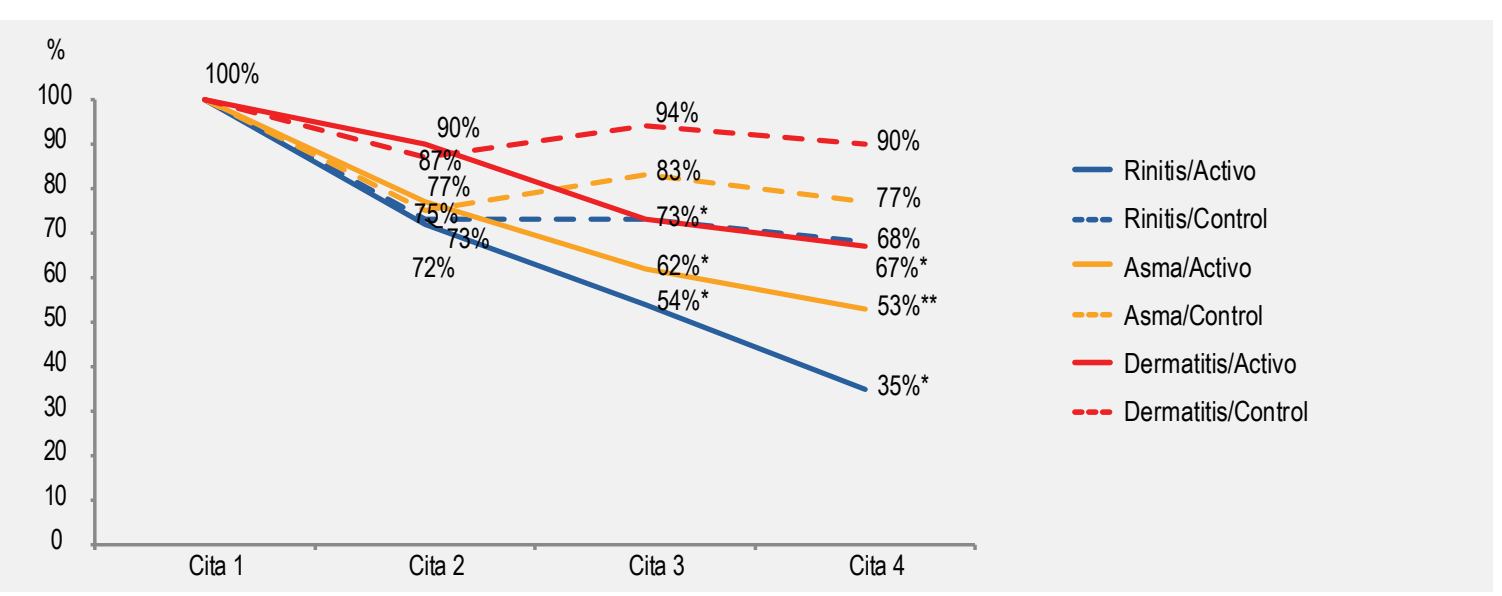

Figura 2. Reducción del tratamiento farmacológico. Cita 1, primera cita; cita 2, 3 a 6 meses; cita 3, 7 a 12 meses; cita 4, 13 a 18 meses. ${ }^{*}<0.05,{ }^{* *}<0.01$.

alérgenos, sin embargo, en algunas sustancias suelen ser insuficientes para controlar completamente los síntomas. ${ }^{12,13}$ Cuando los mecanismos causantes de los síntomas tienen una mediación IgE, la inmunoterapia con alérgenos consigue mejorar el control de los síntomas de los pacientes atópicos y genera tolerancia inmunológica con efecto a largo plazo, ${ }^{14-17}$ previniendo el progreso en la gravedad de los síntomas y el desarrollo de nuevas alergias.

Una dificultad para determinar el éxito de la inmunoterapia es el consecuente costo adicional, sin embargo, los resultados de este estudio muestran que el costo extra inicial suele ser compensado luego de 9 a 12 meses por el mejor control del asma, la rinitis y la dermatitis, que permite una reducción sostenida de los medicamentos.
Aunque el estudio de costos de un tratamiento para una enfermedad determinada debería hacerse en pacientes con solo una enfermedad, la presencia de varias enfermedades tipo IgE mediadas es muy común en los pacientes alérgicos y es infrecuente encontrar pacientes con solo dermatitis, rinitis o asma. Por eso, los análisis realizados en este estudio incluyen la evaluación de costos del tratamiento tanto por cada enfermedad como por paciente, ya que cada uno puede tener diferentes combinaciones de estas enfermedades y, por tanto, diferentes tipos y costos de tratamiento.

Teniendo en cuenta que estas enfermedades suelen ser crónicas, la reducción de la farmacoterapia secundaria a un buen control sintomático puede generar importantes ahorros para el sistema de sa-

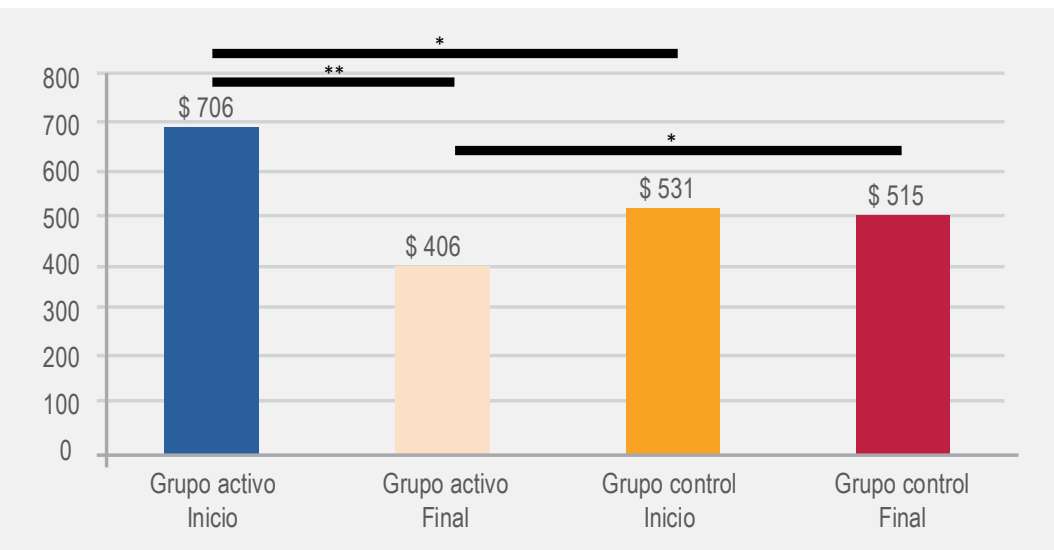

http://www.revistaalergia.mx
Figura 3. Costo global del tratamiento. ${ }^{*}<0.05,{ }^{* *}<0.01$. 
lud y el paciente. En el asma, su alta prevalencia ${ }^{18}$ la hace una enfermedad de alto costo tanto por los medicamentos de control como por las visitas a urgencias. Debido a que 60 a $80 \%$ de los pacientes con asma padece rinitis, los costos del tratamiento se incrementan y se dificulta el control de los síntomas. ${ }^{19}$ Las alergias respiratorias están asociadas con ausentismo laboral o escolar, déficit de atención $\mathrm{y}$, en el caso del asma, muerte por exacerbaciones no controladas. ${ }^{20,21}$ Junto al impacto psicosocial, el ausentismo, las hospitalizaciones, la asistencia a urgencias y otros procesos secundarios generan una importante carga económica para el paciente y el sistema de salud. ${ }^{22}$

En otras investigaciones se ha observado que después de un año de tratamiento, los pacientes presentan un mejor control de los síntomas, lo que permite reducir con pocas recaídas la farmacoterapia hasta en 30 a $45 \%{ }^{23-26}$ En forma similar a nuestra investigación, esos estudios indican que aun cuando en los primeros meses el costo del tratamiento para asma o rinitis aumenta por la inmunoterapia, a mediano plazo este costo se reduce significativamente, incluso, el costo llega a ser menor que en los pacientes que solo reciben el tratamiento farmacológico. Es importante anotar que esta reducción es secundaria al mejor control de los síntomas, lo que potencialmente implica menor número de asistencias a urgencias y hospitalizaciones, mejoría en la calidad de vida de los pacientes y menor riesgo de efectos adversos por la farmacoterapia.

Algunos estudios han demostrado que la aplicación de la inmunoterapia en los niños con rinitis alérgica reduce significativamente el riesgo de desarro- llar posteriormente asma o conjuntivitis, por lo que en ellos la inmunoterapia es activa y preventiva, reduciendo los costos socioeconómicos secundarios. ${ }^{3}$ Igualmente, para conseguir el mejor éxito es necesario seleccionar adecuadamente a los pacientes y que el tratamiento sea aplicado por personal experto.

La dermatitis atópica afecta a $5 \%$ de la población infantil y a 1 de cada 100 adultos. Puede afectar severamente y en ocasiones de manera irreversible la piel, por lo que entre las enfermedades alérgicas es la que más se relaciona con trastornos psicológicos, baja autoestima, depresión e ideación suicida. ${ }^{27}$ Además, se ha observado que los niños menores de 2 años de edad con dermatitis atópica tienen un riesgo 6 a 8 veces mayor de desarrollar asma y otras alergias. Actualmente, varias guías sobre dermatitis han incluido la inmunoterapia como una herramienta en el manejo de esta enfermedad con base en estudios controlados que señalan que su uso en los pacientes con dermatitis moderada o severa disminuye el requerimiento de medicamentos tópicos y sistémicos, cuyo empleo crónico frecuentemente ocasiona efectos adversos. ${ }^{14,28,29}$

Los resultados de esta investigación muestran que la inmunoterapia genera un costo adicional importante en el tratamiento de la dermatitis, sin embargo, al igual que en el asma y la rinitis, el costo se reduce significativamente entre los 9 y 12 meses y el gasto total es similar al del grupo control.

Aunque al evaluar por separado el gasto para la rinitis y la dermatitis, los grupos control tuvieron un gasto levemente inferior que los grupos activos en la última cita, la mayoría de los pacientes con dermatitis, al igual que buena parte de aquellos con rinitis,

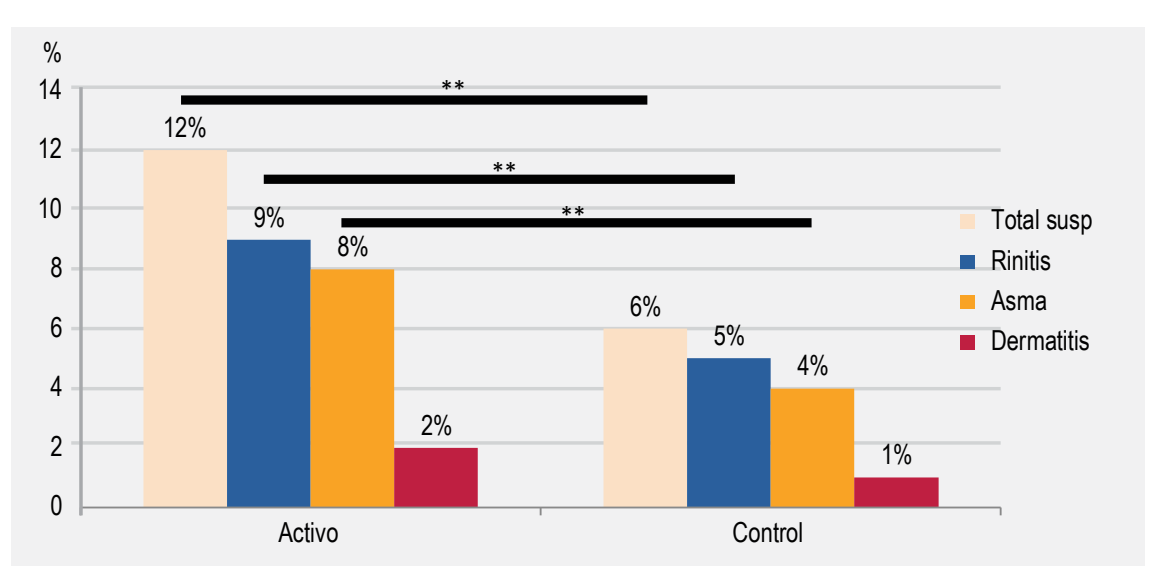

Figura 4. Pacientes con suspensión de medicamentos al final del estudio. Total susp, pacientes con suspensión total de la farmacoterapia; \%, pacientes independientemente de la enfermedad de base. ${ }^{* *}<0.01,{ }^{*}<0.05$. 
tenía varias enfermedades alérgicas y al evaluar el costo global del tratamiento en el grupo activo hubo mayor ahorro, el cual fue sostenido en el grupo de pacientes con inmunoterapia, ya que una menor cantidad requirió aumento de dosis en comparación con el grupo control y mayor proporción suspendió completamente la farmacoterapia.

Nuestro estudio tiene algunas debilidades propias de los análisis retrospectivos: para evaluar el control clínico hubiera sido adecuado utilizar una escala unificada de control de síntomas para cada enfermedad, sin embargo, el monitoreo de la dosis administrada de farmacoterapia es un parámetro frecuentemente utilizado para evaluar la mejoría clínica.

Otro aspecto fue que al analizar los datos de un solo centro de salud especializado, la mayoría de los pacientes atendidos padecía cuadros severos o de difícil control, lo cual se reflejó en un costo mayor del tratamiento en comparación con los pacientes con síntomas leves atendidos en centros de atención primaria, por lo que los resultados hubieran sido más sólidos si el estudio hubiera sido multicéntrico. No obstante, la población con síntomas más severos presenta una oportunidad: son precisamente los pacientes con cuadros severos los que más se pueden beneficiar de la inmunoterapia y quienes incurren en mayores gastos económicos.

Debido a las limitaciones en la fuente de información, los gastos en citas a urgencias no pudieron ser evaluadas, pero hubo menor reporte de exacerbaciones que requirieron urgencias en las historias clínicas de los pacientes con asma que recibían in- munoterapia (datos no mostrados), lo que hace pensar que además de menor necesidad de tratamiento farmacológico, la inmunoterapia puede tener un impacto importante en reducir las exacerbaciones.

Por al amplio surtido de medicamentos y marcas actualmente disponibles para el manejo de las enfermedades estudiadas, el valor asignado fue obtenido del promedio de los precios para las marcas más usadas de cada molécula. Esta medida limita proporcionar el costo exacto del tratamiento en cada paciente, pero al ser utilizado el mismo parámetro en los grupos control y activo para todos los medicamentos no afectó la evaluación de las diferencias observadas.

En conclusión, estos resultados - al igual que los obtenidos sobre eficacia en estudios nacionales e internacionales - sustentan a la inmunoterapia como clínicamente efectiva y segura y que luego de un año reduce sustancialmente el costo global del tratamiento de las enfermedades alérgicas severas.

No obstante lo anterior, es necesario realizar estudios preferentemente prospectivos y multicéntricos que incluyan pacientes con síntomas leves para confirmar estos resultados y caracterizar a la población blanco.

\section{Conflicto de intereses}

El presente artículo fue financiado por los autores.

\section{Agradecimientos}

A la doctora Elizabeth López por su apoyo en la recolección de los datos.

\section{Referencias}

1. Sawicki GS, Vilk Y, Schatz M, Kleinman K, Abrams A, Madden J. Uncontrolled asthma in a commercially insured population from 2002 to 2007: trends, predictors, and costs. J Asthma. 2010;47(5):574-80. doi: 10.3109/0277090100379284

2. Möller C, Dreborg S, Ferdousi HA, Halken S, Høst A, Jacobsen L, et al. Pollen immunotherapy reduces the development of asthma in children with seasonal rhinoconjunctivitis (the PAT-study). J Allergy Clin Immunol. 2002;109(2):251-6. doi: 10.1067/mai.2002.121317

3. Jacobsen L, Niggemann B, Dreborg S, Ferdousi HA, Halken S, Høst A, et al. Specific immunotherapy has long-term preventive effect of seasonal and perennial asthma: 10-year follow-up on the PAT Study. Allergy. 2007;62(8):943-8. doi: 10.1111/j.1398-9995.2007.01451.x

4. Abramson MJ, Puy RM, Weiner JM. Injection allergen immunotherapy for asthma. Cochrane Database Syst Rev. 2010(8):CD001186.

5. Bousquet J, Lockey R, Malling HJ. Allergen immunotherapy: therapeutic vaccines for allergic diseases. A WHO position paper. J Allergy Clin Immunol. 1998;102(4 Pt 1):558-62. doi: 10.1002/14651858. 
6. Simons FE, Ardusso LR, Bilò MB, El-Gamal YM, Ledford DK, Ring J, et al. World Allergy Organization anaphylaxis guidelines: summary. J Allergy Clin Immunol. 2011;127(3):587-93.e1-22. doi: 10.1016/j. jaci.2011.01.038.

7. Fernández-Caldas E, Baena-Cagnani CE, López M, Patiño C, Neffen HE, Sánchez-Medina M, et al. Cutaneous sensitivity to six mite species in asthmatic patients from five Latin American countries. J Investig Allergol Clin Immunol. 1993;3(5):245-9.

8. Sánchez J, Diez S, Cardona R. Sensibilización a aeroalergenos en pacientes alérgicos de Medellín, Colombia. Rev Alergia Mex. 2012;59(3):139-47.

9. Sánchez J, Diez S, Cardona R. Frecuencia de sensibilización a animales en un área tropical. Rev Alerg Mex. 2014;61(2):81-9.

10. Sánchez J, Restrepo M, Diez S, Cardona R. Comparación del efecto clínico de la inmunoterapia en pacientes con asma alérgica según la edad y el patrón de sensibilización. Alerg Asma Inmunol Pediatr. 2012;21(3):77-85.

11. Charlson ME, Pompei P, Ales KL, MacKenzie CR. A new method of classifying prognostic comorbidity in longitudinal studies: development and validation. J Chronic Dis. 1987;40(5):373-83. doi:10.1016/00219681(87)90171-8

12. van Strien RT, Koopman LP, Kerkhof M, Spithoven J, de Jongste JC, Gerritsen J, et al. Mite and pet allergen levels in homes of children born to allergic and nonallergic parents: the PIAMA Study. Environ Health Perspect. 2002;110(11):A693-8.

13. Caudri D, Wijga AH, Smit HA, Koppelman GH, Kerkhof M, Hoekstra MO, et al. Asthma symptoms and medication in the PIAMA birth cohort: evidence for under and overtreatment. Pediatr Allergy Immunol. 2011;22(7):652-9. doi: 10.1111/j.1399-3038.2011.01193.x

14. Sánchez-Caraballo J, Cardona-Villa R. Clinical and Immunological changes of Immunotherapy in patients with atopic dermatitis: randomized controlled trial. ISRN Allergy. 2012:1-9. doi: 10.5402/2012/183983

15. Jutel $M$, Akdis CA. Immunological mechanisms of allergen-specific immunotherapy. Allergy. 2011;66(6):725-32. doi: 10.1111/j.1398-9995.2011.02589.x

16. Scadding G, Durham SR. Mechanisms of sublingual immunotherapy. Immunol Allergy Clin North Am. 2011;31(2):191-209, viii. doi: 10.1016/j.iac.2011.02.005

17. Cox L, Nelson H, Lockey R, Calabria C, Chacko T, Finegold I, et al. Allergen immunotherapy: a practice parameter third update. J Allergy Clin Immunol. 2011;127(1 Suppl):S1-55. doi: 10.1016/j.jaci.2010.09.034

18. Dennis RJ, Caraballo L, García E, Rojas MX, Rondon MA, Pérez A, et al. Prevalence of asthma and other allergic conditions in Colombia 2009-2010: a cross-sectional study. BMC Pulm Med. 2012;12:17. doi: 10.1186/1471-2466-12-17

19. Kurukulaaratchy RJ, Karmaus W, Arshad SH. Sex and atopy influences on the natural history of rhinitis. Curr Opin Allergy Clin Immunol. 2012;12(1):7-12. doi: 10.1097/ACI.0b013e32834ecc4e

20. Vergara C, Caraballo L. Asthma mortality in Colombia. Ann Allergy Asthma Immunol. 1998;80(1):55-60. doi:10.1016/S1081-1206(10)62940-7

21. Neffen H, Baena-Cagnani CE, Malka S, Solé D, Sepúlveda R, Caraballo L, et al. Asthma mortality in Latin America. J Investig Allergol Clin Immunol. 1997;7(4):249-53.

22. Rodríguez-Martínez CE, Sossa-Briceño MP, Castro-Rodriguez JA. Cost-utility analysis of the inhaled steroids available in a developing country for the management of pediatric patients with persistent asthma. J Asthma. 2013;50(4):410-8. doi: 10.3109/02770903.2013.767909

23. Hankin CS, Cox L, Bronstone A, Wang Z. Allergy immunotherapy: reduced health care costs in adults and children with allergic rhinitis. J Allergy Clin Immunol. 2013;131(4):1084-91. doi: 10.1016/j. jaci.2012.12.662

24. Canonica GW, Passalacqua G. Disease-modifying effect and economic implications of sublingual immunotherapy. J Allergy Clin Immunol. 2011;127(1):44-5. doi: 10.1016/j.jaci.2010.09.037

25. Bielory L, Mongia A. Current opinion of immunotherapy for ocular allergy. Curr Opin Allergy Clin Immunol. 2002;2(5):447-52. 
Sánchez J Consecuencias económicas de la inmunoterapia

26. Lin SY, Erekosima N, Kim JM, Ramanathan M, Suárez-Cuervo C, Chelladurai Y, et al. Sublingual immunotherapy for the treatment of allergic rhinoconjunctivitis and asthma: a systematic review. JAMA. 2013;309(12):1278-88. doi: 10.1001/jama.2013.204

27. Yaghmaie P, Koudelka CW, Simpson EL. Mental health comorbidity in patients with atopic dermatitis. J Allergy Clin Immunol. 2013;131(2):428-33. doi: 10.1016/j.jaci.2012.10.041

28. Bae JM, Choi YY, Park CO, Chung KY, Lee KH. Efficacy of allergen-specific immunotherapy for atopic dermatitis: A systematic review and meta-analysis of randomized controlled trials. J Allergy Clin Immunol. 2013; 132(1):110-7. doi: 10.1016/j.jaci.2013.02.044

29. Sánchez J, Páez B, Macías A, Olmos C, de Falco A. Atopic dermatitis guideline. Position paper from the Latin American Society of Allergy, Asthma and Immunology. Rev Alerg Mex. 2014;61(3):178-211. 